\title{
Finding Your Way in a Forest: On Different Types of Trees and Their Properties
}

\author{
Igor Walukiewicz ${ }^{\star}$ \\ CNRS LaBRI \\ Université de Bordeaux \\ 351, Cours de la Libération \\ 33405 Talence, France
}

We see trees in almost any part of computer science. Traditionally, ranked trees, that are nothing else but terms, captured most attention, although exceptions could have been found in graph theory or linguistics 9]. Recently unranked trees are a subject of renewed interest, mainly because of the developement of XML 22. It is also quite comon nowadays to see trees with infinite paths, especailly in the context of verification. We will omit this aspect, as for the questions we want to discuss finite trees are sufficiently interesting. We prefer instead to make distinction between ordered and unordered trees, i.e., distinguish situations when syblings are ordered or not. Thus we will deal with four types of trees depending on two parameters: ranked/unranked, and ordered/unordered.

There are even more formalisms to describe tree properties than there are tree types. Here, the main reference point for us will be monadic second-order logic which captures recognizable sets of trees. This logic has a binary predicate interpreted as a descendant relation in a tree and a monadic predicate for every possible node label. If models are ordered trees then the logic may also have another binary predicate interpreted as the sybling order. Important logics are obtained by restricting the range of quantification: when quantifying only over elements we obtain first-order logic (FOL), when quantifying over chians (sets where every pair of nodes is in a descendant relation) we get chain logic, finally antichain logic is obtained when quatifiers range over sets where no two elements are in the descendant relation. Appart from these classical logics we have important variants of modal and temporal logics over trees: CTL, CTL*, PDL, the $\mu$-calculus.

Given this multitude of formalisms, the first question one can ask is to compare their expressive power, i.e., establish if all properties expressible in a logic A can be also expressed in a logic B. We know the answers to this kind of questions for the formalism listed above. Figure 1 presents the case of ranked ordered trees. Some of the presented inclusions are nontrivial. For insatance, the inclusions of the MSOL in the $\mu$-calculus 18 or FOL in CTL* 14 show that one can obtain the same expressive power using very different means. For other types of trees the picture is not the same. For example, if we consider ordered unranked

\footnotetext{
^ Work supported by project DOTS (ANR-06-SETI-003).
} 


$\begin{array}{cccccc} & \mu \text {-calculus } & = & \text { MSOL } & = & \text { antichain logic } \\ \text { UH } & & \text { Ut } & & \text { UH } \\ \text { CTL } \subsetneq \quad \text { CTL }^{*} & = & \text { FOL } & f & \text { chain logic }\end{array}$

Fig. 1. Relations between logics over ordered binary trees

trees, all equalities on the left change to strict inclusions, and chain logic becomes incomparable with antichaing logic.

While diagrams as that on Figure 1 are important, they are far from giving a complete explanation of the expressive power of the logics in question. Consider the following situation. Separating FOL from MSOL over ordered binary trees is easy. We know that over words FOL cannot express counting modulo properties [20]. For example, "even length" is not first order definable. A short argument shows that the language of trees with the leftmost path of even length is not FOL expressible. This observation though, does not tell much about what properties are expressible in FOL. Take the language of ordered binary trees "the depths of all leaves are even". Somehow surprisingly, it turns out that this language is FOL-expressible 19. Indeed it is a major open question to find an algorithm deciding if a given regular tree language is experssible in FOL. At present decidable characterisations are known for very few fragments of MSOL [1748].

Apart from the mathematical curiosity, there is a growing number of reasons for looking closer at tree formalisms. In the context of XML, the aspect of data (infnite set of labels with some operations on them) is important. It is rather difficult to come with a decidable nontrivial formalism [6]16]. Understanding well expressive power in the case without data can help substantially.

Another reason is the study of order invariance. A property is order invariant if does not distingush two trees that differ only in the order of syblings. It is natural to ask if such a property can be expressed without referring to this order. Over unranked trees, order invariant properties are exactly those expressible in MSOL extended with counting modulo quantifiers 11. The language "the depths of all leaves are even" described above cannot be defined in FOL without a sybling order. So the situation is much less clear for FOL. Curiously enough if we restrict to FOL[succ] where we allow only successor relation in place of descendant relation then all order invariant FOL[succ] properites can be expressed in FOL[succ] [2].

Finally, there is a question of automata and grammars for trees. In the literature one can find many proposals of differen automata on trees. Even for ordered binary trees we have for example several versions of tree-walking automata 12513. For other types of trees the number of variants is even bigger [10]. The similar situation is with regular expressions for trees [2115173]. Better understanding of logical formalisms is indenspensable to classify and clarify all these notions.

In this talk we will survey some recent results in the field. We will start with a unifying presentation of formalisms discussed above. For this a small detour to algebra will be useful [8]. We will present dependencies between different 
formalisms, and known decidability results. Some less common questions as order invariance, or existance of a finite base will be also considered. The talk will present joint work with Mikolaj Bojańczyk.

\section{References}

1. Benedikt, M., Segoufin, L.: Regular languages definable in FO. In: Diekert, V., Durand, B. (eds.) STACS 2005. LNCS, vol. 3404, pp. 327-339. Springer, Heidelberg (2005)

2. Benedikt, M., Segoufin, L.: Towards a characterization of order-invariant queries over tame structures. In: Ong, L. (ed.) CSL 2005. LNCS, vol. 3634, pp. 276-291. Springer, Heidelberg (2005)

3. Bojanczyk, M.: Forest expressions. In: Duparc, J., Henzinger, T.A. (eds.) CSL 2007. LNCS, vol. 4646, pp. 146-160. Springer, Heidelberg (2007)

4. Bojanczyk, M.: Two-way unary temporal logic over trees. In: LICS 2007, pp. 121130 (2007)

5. Bojanczyk, M., Colcombet, T.: Tree-walking automata do not recognize all regular languages. In: STOC 2005, pp. 234-243. ACM, New York (2005)

6. Bojanczyk, M., David, C., Muscholl, A., Schwentick, T., Segoufin, L.: Two-variable logic on data trees and XML reasoning. In: PODS 2006, pp. 10-19. ACM, New York (2006)

7. Bojanczyk, M., Walukiewicz, I.: Characterizing EF and EX tree logics. Theoretical Computer Science 358(2-3), 255-272 (2006)

8. Bojanczyk, M., Walukiewicz, I.: Forest algebras. In: Flum, J., Grädel, E., Wilke, T. (eds.) Logic and Automata. Texts in Logic and Games, vol. 2, pp. 107-132. Amsterdam University Press (2007)

9. Carpenter, B.: The Logic of Typed Future Structures. Cambridge University Press, Cambridge (1992)

10. Comon, H., Dauchet, M., Gilleron, R., Lugiez, F.J.D., Tison, S., Tommasi, M.: Tree automata techniques and applications (2002), http://www.grappa.univ-lille3.fr/tata/

11. Courcelle, B.: The monadic second-order logic of graphs V: On closing the gap between definability and recognizability. Theor. Comput. Sci. 80(2), 153-202 (1991)

12. Engelfriet, J., Hoogeboom, H.J.: Tree-walking pebble automata. In: Karhumäki, J., et al. (eds.) Jewels are forever, pp. 72-83. Springer, Heidelberg (1999)

13. Engelfriet, J., Hoogeboom, H.J., Samwel, B.: XML transformation by tree-walking transducers with invisible pebbles. In: PODS 2007, pp. 63-72. ACM, New York (2007)

14. Hafer, T., Thomas, W.: Computation tree logic CTL* and path quantifiers in the monadic theory of the binary tree. In: Ottmann, T. (ed.) ICALP 1987. LNCS, vol. 267, pp. 269-279. Springer, Heidelberg (1987)

15. Heuter, U.: First-order properties of trees, star-free expressions, and aperiodicity. In: Cori, R., Wirsing, M. (eds.) STACS 1988. LNCS, vol. 294, pp. 136-148. Springer, Heidelberg (1988)

16. Jurdzinski, M., Lazic, R.: Alternation-free modal mu-calculus for data trees. In: LICS 2007, pp. 131-140. IEEE Computer Society Press, Los Alamitos (2007)

17. Martens, W., Neven, F., Schwentick, T., Bex, G.J.: Expressiveness and complexity of XML schema. ACM Trans. Database Syst. 31(3), 770-813 (2006)

18. Niwiński, D.: Fixed points vs. infinite generation. In: LICS 1988, pp. 402-409 (1988) 
19. Potthoff, A.: First-order logic on finite trees. In: Mosses, P.D., Schwartzbach, M.I., Nielsen, M. (eds.) TAPSOFT 1995. LNCS, vol. 915, pp. 125-139. Springer, Heidelberg (1995)

20. Schützenberger, M.P.: On finite monoids having only trivial subgroups. Information and Control 8, 190-194 (1965)

21. Thomas, W.: Logical aspects in the study of tree languages. In: Paredaens, J. (ed.) ICALP 1984. LNCS, vol. 172, pp. 31-50. Springer, Heidelberg (1984)

22. Vianu, V.: A web odyssey: From CODD to XML. In: PODS 2001, ACM, New York (2001) 